\title{
Dengue Fever in Iran: A Case Report
}

\author{
Masoud Ebrahimi, ${ }^{1}$ Alireza Abadi, ${ }^{2,}{ }^{*}$ Haniyeh Bashizadeh-Fakhar, ${ }^{3}$ and Elaheh Fahimi ${ }^{4}$ \\ ${ }^{1}$ Department of Infectious Diseases, Faculty of Medicine, Sabzevar University of Medical Sciences, Sabzevar, IR Iran \\ ${ }^{2}$ Faculty of Medicine, Sabzevar University of Medical Sciences, Sabzevar, IR Iran \\ ${ }^{3}$ Shahid Beheshti University of Medical Sciences, Tehran, IR Iran \\ ${ }^{4}$ Faculty of Medicine, Sabzevar University of Medical Sciences, Sabzevar, IR Iran \\ "Corespounding author: Alireza Abadi, Faculty of Medicine, Sabzevar University of Medical Sciences, Sabzevar, IR Iran. E-mail: haniyehfakhar@yahoo.com
}

Received 2016 December 06; Accepted 2016 December 11.

\begin{abstract}
A young woman with symptoms similar to common cold was referred to Vasei hospital of Sabzevar. The patient had travelled to Malaysia which was mentioned in her history. She hospitalized with symptoms of high fever, headache, and lethargy. Then, she underwent supportive therapy. In next day, the symptoms such as bone pain, myalgias, shortness of breath, abdominal pain, severe headache, eye strain and maculopapular lesions along with nausea, vomiting, and diarrhea were appeared. Laboratory findings showed high grade fever, leukopenia, and severe thrombocytopenia. Considering the findings, dengue fever was diagnosed and the patient serum sample was sent to a reference laboratory for presence of Immunoglobulin M (IgM). The aim of this study was to introduce a new case of dengue fever in Iran.
\end{abstract}

Keywords: Dengue Fever, Dengue Hemorrhagic Fever, Thrombocytopenia

\section{Introduction}

Dengue fever or Break bone fever is common in tropical zones of Asia (1). Transmitted by arthropods, dengue fever was introduced as the most important viral disease in 2012 (2). Annually, 50 to 100 million dengue infections occur in the world (3). The infection is transmitted by mosquitoes called Aedes Aegypti and Aedes Albopictus and has 4 serotypes; DEN1, DEN2, DEN3, and DEN4 (4). Infection is caused by a Flavi virus (5) through mosquito bite. The incubation period is 4 - 7 days after infection (4). The symptoms may vary from mild to hemorrhagic fever to dengue shock syndrome (DSS). Mortality rate due to dengue has been different across the globe, reported 10\% - 15\% in some areas, and less than $1 \%$ elsewhere (6). Dengue fever is a viral disease characterized by fever, headache, aching bones and joints, myalgias and rash (7). WHO has identified several criteria for dengue diagnosis based on symptoms and laboratory findings; people living in endemic areas or those who travel to such areas with likelihood of dengue, manifest two of the followings can be suspected of this fever: nausea, vomiting, rash, pain, positive tourniquet test, leukopenia and warning signs including abdominal pain, tenderness, persistent vomiting, fluid retention, mucosal bleeding, lethargy and irritability, enlarged liver more than $2 \mathrm{~cm}$ and elevated hematocrit with rapid decrease in platelets (8). The purpose of this paper was to present a case with similar symptoms of dengue fever in Iran along with providing information on its diagnosis.

\section{Case Presentation}

The patient was a 39-year-old woman who had lived in Kuala Lumpur, Malaysia for 6 years. Once she came to Iran with her son, during the flight, her son showed fever and headache without any other specific symptoms. After some days, they referred to a GE in Tehran but no improvement was seen in the patient. They then went to Sabzevar city where the patient was hospitalized with 38.1 grade fever, headache and mild rash and underwent normal saline solution and acetaminophen treatment.

She showed bodily pain, nausea, lethargy, vomiting blood and bone pain and myalgia with severity of $6-7$ from 10, (score: 6 - 7) three days after return.

Severe headache, eye strain, and drooping eyelids with mucoid diarrhea were also observed a few days after hospitalization. The next day, maculopapular lesions (Figure 1) appeared all over her body with abdominal pain and tenderness in RUQ, shortness of breath, orthopnea, and dry cough. However, no mucocutaneous bleeding, hematuria, gastrointestinal bleeding and epistaxis were observed. In the hospital, the patient got urinary tract infection (UTI).

Laboratory findings are presented in Table 1. Results of abdominal sonography, chest x-ray and EKG were normal. Serum sample of the patient with diagnosis of dengue fever was sent to a reference laboratory where the diagnosis was affirmed positive (l gm in serum). 
Table 1. Vital Signs and Laboratory Parameters of Patient During Hospitalization

\begin{tabular}{|c|c|c|c|c|}
\hline Clinical and Laboratory Parameters & At the Time of Admission & 2nd Day & 3rd Day & 4th Day \\
\hline Temperature, $\mathbf{F}$ & 38.1 & 38 & 37.8 & 37.2 \\
\hline Pulse rate, per min & 121 & 88 & 83 & 80 \\
\hline Blood pressure, $\mathrm{mmHg}$ & $110 / 80$ & $120 / 80$ & $120 / 80$ & $120 / 80$ \\
\hline Total leucocytes count, per cc3 & 1.4 & 1.5 & 2 & 3.2 \\
\hline Thrombocyte count, per $\mathrm{mm}^{3}$ & 73 & 77 & 46 & 66 \\
\hline Haematocrit, \% & 31 & 31.3 & 32.8 & 34.7 \\
\hline ALT, IU/L & 22 & - & - & - \\
\hline AST, IU/L & 51 & - & - & - \\
\hline Prothrombin time, $s$ & 12 & - & - & - \\
\hline Partial thromboplastin time & 26 & - & - & - \\
\hline International normalised ratio & 1 & - & - & - \\
\hline ESR & - & - & 14 & - \\
\hline CRP & Negative & & & \\
\hline Wright & Negative & & & \\
\hline PBS & Normal & & & \\
\hline
\end{tabular}

Abbreviations: ALT, alanine transaminase, AST, aspartate transaminase, CRP, C reactive protein, ESR, erythrocyte sedimentation rate, PBS, peripheral blood smear.

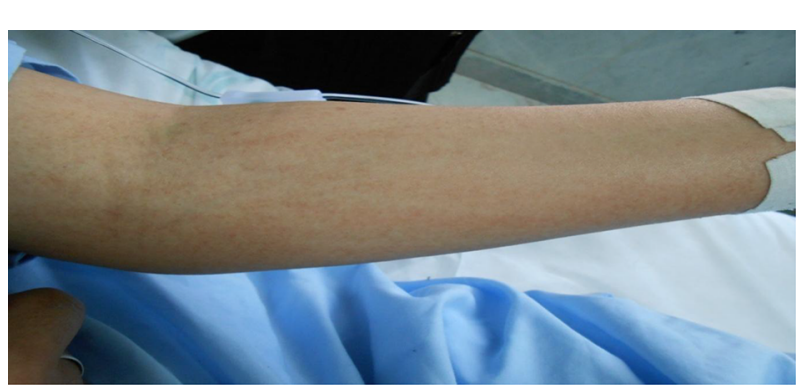

Figure 1. Maculopapular Lesions

\section{Discussion}

Dengue fever is a viral disease transmitted by mosquitoes. The disease severity varies from mild to hemorrhagic fever and dengue shock syndrome. It has 3 phases after the sudden onset of the fever, which includes fever phase, the acute phase, and recovery phase. Thrombocytopenia is witnessed in acute phase. The incubation period usually lasts from 4 to 7 days and 8 to 10 days after infection during which the virus can be transmitted by mosquito bite. The disease is endemic in tropical and sub-tropical areas where 50.000.000 new occurrences are being reported every year. About 24,000 deaths due to dengue have been recorded in the past four decades.

Symptoms manifested as fever, headache, muscle aches, sometimes rash, and mouth, nose and gastrointestinal bleeding which may even lead to death. But they gradually disappear within 2 weeks. WHO has in fact identified criteria (mentioned in Introduction) for diagnosis of dengue fever which are based on clinical symptoms and laboratory findings.

In this case report, according to WHO criteria, a person who had Symptoms such as fever, headache, muscle aches, nausea, lethargy, vomiting, tenderness in right upper quadrant (RUQ), maculopapular lesions, shortness of breath, dry cough without mucosal and gastrointestinal bleeding, came from Kuala Lumpur city to Iran. However, this disease can have very different manifestations which are not included in WHO criteria. As a case reported by Thushara matthisaet al. who had been referred with heart rhythm disorder in bleeding phase in addition to WHO dengue criteria without any previous history of myocarditis (9).

In a study by Verma et al. a 40-year-old man with complaints of fever, myalgia, weakness, and loss of limbs was reported. Thus, the disease symptoms can manifest as neurological disorders. The atypical manifestations include quadriplegia, seizure, headache, aseptic meningitis, encephalitis, mononeuropathy and Guillain-Barre (10). Other manifestations found in dengue can be renal dysfunction as reported by Vasanwala et al. in which a 42-year-old man with muscle aches, fever, thrombocytopenia, petechiae, 
and decreased serum albumin was diagnosed. His urine protein level was at nephrotic syndrome with hematuria and Cast (5). Moreover, the disease may be accompanied by a variety of other symptoms including acute abdomen. In a case reported by Al-Araimi et al. a 38-year-old woman had been referred with fever, abdominal pain, diarrhea and vomiting (11). Examinations only showed generalized tenderness without abdomen guarding and other things have been reported normal in examinations. Through performing other examinations and laboratory tests in addition to WHO criteria, the patient was suspected of dengue fever. After serological evaluation, l gm was positive.

Furthermore, dengue disease may manifest with gastrointestinal symptoms which may make diagnosis and treatment difficult and wrong. Thus, this disease should be considered in differential diagnosis of acute abdomen. Based on reported cases in different countries, it can be said that dengue can appear anywhere in the world with diverse and deadly symptoms. As time goes by, the world is facing warmer climate, more draught and stagnant waters, all being good conditions for mosquito breeding, thus a better understanding of this fever seems necessary.

\subsection{Conclusions}

Regarding the above mentioned points, the WHO motto "Small bite, big threat!" is now being somehow realized. Iran has a hot and dry climate at which many dengue transmitter mosquitoes can live for example, stagnant and dirty water in car wheels and flower pots, etc. Therefore, dengue can be considered as a treat in Iran; complete recognition and early diagnosis can be helpful in promoting public health and reducing dengue treatment costs.

\section{Acknowledgments}

I appreciated Dr Masoud Ebrahimi and Dr Hanieh Bashi Zadeh Fakhar for help us to complete our research and also, thank to all the other people and staff in hospital and at university.

\section{Footnotes}

Authors' Contribution: Alireza Abadi was executor of the article. Masoud Ebrahimi introduced case report and edited the article, and Alireza Abadi was author and presenter of the article. Haniyeh Bashizadeh-Fakhar submitted and edited the article. Elaheh Fahimi wrote and edited the article.

Conflict of Interest: There is no conflict of interest in this research.

Funding/Support: Sabzevar University of Medical Sciences.

\section{References}

1. Deen JL, Harris E, Wills B, Balmaseda A, Hammond SN, Rocha C, et al. The WHO dengue classification and case definitions: time for a reassessment. Lancet. 2006;368(9530):170-3. doi: 10.1016/S01406736(06)69006-5. [PubMed: 16829301].

2. World Health Organization . Global Strategy For Dengue prevention and Control, 2010-2020. Vector Ecology and Management Unit Department of Control of Neglected Tropical Diseases. ; 2012.

3. Gubler DJ. The global emergence/resurgence of arboviral diseases as public health problems. Arch Med Res. 2002;33(4):330-42. [PubMed: 12234522].

4. Jelinek T. Dengue fever in international travelers. Clin Infect Dis. 2000;31(1):144-7. doi: 10.1086/313889. [PubMed: 10913412].

5. Vasanwala FF, Puvanendran R, Ng JM, Suhail SM. Two cases of selflimiting nephropathies secondary to dengue haemorrhagic fever. Singapore Med J. 2009;50(7):e253-5. [PubMed:19644612].

6. Swaminathan S, Khanna N. Dengue vaccine-current progress and challenges. Curr Sci. 2010;98:369-78.

7. Kumar J, Kumar A, Gupta S, Jain D. Dengue haemorrhagic fever: An unusual cause of intracranial haemorrhage. J Neurol Neurosurg Psychiatr. 2007;78(3):253.

8. World Health Organization . Dengue guidelinesfor diagnosis, treatment, prevention and Control. Geneva: World Health Organization; 2009.

9. Matthias AT, Indrakumar J, Gunatilake SB. Ventricular trigeminy in a patient with serologically confirmed dengue haemorrhagic fever. Int Arch Med. 2014;7:28. doi: 10.1186/1755-7682-7-28. [PubMed: 24904689].

10. Verma SP, Himanshu D, Tripathi AK, Vaish AK, Jain N. An atypical case of dengue haemorrhagic fever presenting as quadriparesis due to compressive myelopathy. BMJ Case Rep. 2011;2011 doi: 10.1136/bcr.10.2010.3421. [PubMed: 22700077].

11. Al-Araimi H, Al-Jabri A, Mehmoud A, Al-Abri S. Dengue Haemorrhagic Fever presenting as Acute Abdomen. Sultan Qaboos Univ Med J. 2011;11(2):265-8. [PubMed: 21969900]. 